\title{
Time-Orthogonal Unitary Dilations and Noncommutative Feynman-Kac Formulae. II"
}

\author{
By \\ R. L. HUdSON*, P. D. F. ION** and K. R. PARTHASARATHY***
}

\begin{abstract}
In a previous paper [6] it was shown that a certain two-parameter dilation of a given strongly continuous self-adjoint contraction semigroup, called the time-orthogonal unitary dilation, gives rise to noncommutative Feynman-Kac formulae through the mechanism of Boson second quantisation in Fock space. This paper explores the modifications of this theory which arise firstly by using Fermion rather than Boson second quantisation, and secondly by using Boson second quantisation based on extremal universally invariant states of the CCR algebra. In the second case it is found that the programme is successful if and only if the infinitesimal generator of the original semigroup is of trace class.
\end{abstract}

\section{§1. Introduction}

In a previous paper [6] a certain two-parameter unitary evolution, called the time-orthogonal unitary dilation, was constructed for a given strongly continuous self-adjoint contraction one-parameter semigroup. Upon passing to second quantisations in Boson Fock space, one obtains a unitary evolution possessing properties of Euclidean covariance and independence which permit the construction of a non-commutative Feynman-Kac formula $[5,6]$ for perturbations of the semigroup which is the second quantisation of the originally

Communicated by H. Araki, May 31, 1983.

* Mathematics Department, University of Nottingham, University Park, Nottingham NG 7 2RD, England.

** Mathematical Reviews, University of Michigan, 611 Church Street, P. O. Box 8604, Ann Arbor, Michigan 48107, USA.

*** Indian Statistical Institute, 7, S. J. S. Sansanwal Marg, New Delhi-110016, India.

1) Parts of this work were carried out when the first and second authors were participants in the Sonderforschungsbereich 123 at the University of Heidelberg (Stochastische Mathematische Modelle) of the Deutsche-Forschungsgemeinschaft and when the third author was Visiting Fellow at the University of Warwick. 
given contraction semigroup.

The purpose of the present work is to investigate the modifications of this theory which arise when Fock Boson second quantisation is replaced, firstly by Fermion second quantisation in Fock space, and secondly by non-Fock second quantisation based on extremal universally invariant states of the CCR algebra.

We recall that in [6], a non-commutative Feynman-Kac formula was associated with the following structure. A reflective covariant system $\left(N,\left(N_{s, t}\right),\left(\gamma_{t}\right), \rho\right)$ consists of a von Neumann algebra $N$, a doubly filtering system $\left(N_{s, t}, s>t\right)$ of von Neumann subalgebras of $N$, and a realisation of the one-dimensional Euclidean group by automorphisms of $N$ in which for $t \in \boldsymbol{R}$ translation through $t$ is realised by $\gamma_{t}$ and reflection at the origin by $\rho$, satisfying the conditions

$$
\begin{gathered}
\gamma_{r}\left(N_{s, t}\right) \subseteq N_{r+s, r+t}, \\
\rho N_{s, t} \subseteq N_{-t,-s}
\end{gathered}
$$

for $r, s, t \in \boldsymbol{R}, s \geq t$. A reflective covariant reducing map $j$ for such a system is a strongly continuous linear map from $N$ to the algebra $B\left(\mathfrak{G}_{0}\right)$ of bounded operators in a Hilbert space $\mathfrak{h}_{0}$ satisfying the conditions

$$
\begin{gathered}
j\left(A^{*}\right)=j(A)^{*}, \\
j\left(A_{1} A_{2}\right)=j\left(A_{1}\right) j\left(A_{2}\right), \\
j \circ \gamma_{t}=j \circ \rho=j
\end{gathered}
$$

for arbitrary $A \in N, t \in \boldsymbol{R}$ and $A_{1}, A_{2}$ belonging to subalgebras $N_{s_{1}, t_{1}}$, $N_{s_{2}, t_{2}}$ for which the time intervals $\left.\left.\left.] t_{1}, s_{1}\right],\right] t_{2}, s_{2}\right]$ are disjoint. An evolution $\left(U_{s, t}: s \geq t\right)$ is said to be reflectively covariantly adapted to the system $\left(N,\left(N_{s, t}\right),\left(\gamma_{t}\right), \rho\right)$ if each $U_{s, t} \in N_{s, t}$ and for arbitrary $r, s$, $t \in \boldsymbol{R}$ with $s \geq t$,

$$
\gamma_{r}\left(U_{s, t}\right)=U_{r+s, r+t}, \quad \rho\left(U_{s, t}^{*}\right)=U_{-t,-s} .
$$

Given a reflective covariant reducing map $j$ and a reflectively covariantly adapted evolution $\left(U_{s, t}\right)$ for the system $\left(N,\left(N_{s, t}\right),\left(\gamma_{t}\right)\right.$, $\rho)$, the operators $j\left(U_{s, t}\right)$ form a strongly continuous self-adjoint contraction semigroup in $\mathfrak{H}_{0}$, called the reduced evolution,

$$
j\left(U_{s, t}\right)=e^{-(s-t) H} .
$$

Moreover, if $\left(U_{s, t}\right)$ is unitary, for each self-adjoint $B \in \bigcap_{s \geq t} N_{s, t}$ invariant under the automorphisms $\gamma_{t}, t \in \boldsymbol{R}$ and $\rho$, there is a unique 
cocycle $\left(M_{s, t}^{B}\right)$ for $\left(U_{s, t}\right)$ satisfying

$$
\partial_{t} M_{s, t}^{B}=-M_{s, t}^{B} U_{s, t} B U_{s, t}^{-1}, \quad M_{t, t}^{B}=0
$$

so that in the case when the operators $U_{s, t} B U_{s, t}^{-1} ; s \geq t$, all commute

$$
M_{s, t}^{B}=\exp \left\{-\int_{t}^{s} U_{s, \tau} B U_{s, \tau}^{-1} d \tau\right\} .
$$

The perturbed evolution

$$
U_{s, t}^{B}=M_{s, t}^{B} U_{s, t}
$$

is also reflectively covariantly adapted and the corresponding semigroup is given by

$$
j\left(U_{s, t}^{B}\right)=e^{-(s-t)(H+j(B))} .
$$

Thus in the commuting case we obtain the Feynman-Kac formula

$$
e^{-(s-l)(H+j(B))}=j\left[\exp \left\{-\int_{t}^{s} U_{s, \tau} B U_{s, \tau}^{-1} d \tau\right\} U_{s, t}\right] .
$$

Let $\left(A_{t}: t \geq 0\right)$ be a strongly continuous one parameter self-adjoint contraction semigroup acting in a Hilbert space $\mathfrak{h}_{0}$ and write $A_{t}=$ $\exp (-t H)$ where $H$ is positive and self-adjoint. Let $\mathfrak{h}=L^{2}\left(\boldsymbol{R} ; \mathfrak{h}_{0}\right)$, $\mathfrak{h}_{1}=\mathfrak{h}_{0} \oplus \mathfrak{h}$ and let $J: \zeta \rightarrow(\zeta, 0)$ be the natural embedding of $\mathfrak{h}_{0}$ in $\mathfrak{h}_{1}$. We recall from [6] that there exists a unitary evolution $\left(U_{s, t}: s \geq t\right)$ in $\mathfrak{h}_{1}$, the time-orthogonal unitary dilation of $\left(A_{t}\right)$, such that the following properties for arbitrary $r, s, t \in \boldsymbol{R}$ with $s \geq t$.

a)

$$
A_{s-t}=J^{*} U_{s, t} J
$$

b) in the decomposition $\mathfrak{h}_{1}=\left(\mathfrak{h}_{0} \oplus \mathfrak{h}_{] t, s]}\right) \oplus \mathfrak{h}_{\mathfrak{J t}, s]}^{\perp}$, where $\mathfrak{h}_{] t, s]}$ is the subspace of $\mathfrak{h}$ comprising functions with support $] t, s]$ and $\mathfrak{h}_{j \mathfrak{t}, s]}$ is its orthogonal complement in $\mathfrak{h}, U_{s, t}$ assumes the form $V_{s, t} \oplus I$ where $V_{s, t}$ is a unitary operator in $\mathfrak{h}_{0} \oplus \mathfrak{h}_{1 t, s]}$.

c)

$$
\begin{aligned}
& \left(I \oplus S_{r}\right) U_{s, t}\left(I \oplus S_{r}\right)^{-1}=U_{r+s, r+t}, \\
& (I \oplus R) U_{s, t}^{*}(I \oplus R)^{-1}=U_{-t,-s}
\end{aligned}
$$

where $S_{r}$ and $R$ are the shift and reflection operators in $L^{2}\left(\boldsymbol{R} ; \mathfrak{h}_{0}\right)$

$$
\begin{aligned}
& S_{r} f(t)=f(t-r), \\
& R f(t)=f(-t) .
\end{aligned}
$$

As shown in [6], the second quantisations $\left(\Gamma^{+}\left(U_{s, t}\right), s \geq t\right)$ in Boson Fock space $\Gamma^{+}\left(\mathfrak{h}_{1}\right)=\Gamma^{+}\left(\mathfrak{h}_{0}\right) \otimes \Gamma^{+}(\mathfrak{h})$ form a reflectively covar- 
iantly adapted evolution and the map $j^{+}: T \rightarrow \Gamma^{+}\left(J^{*}\right) T \Gamma^{+}(J)$ from $B\left(\Gamma^{+}\left(\mathfrak{G}_{1}\right)\right)$ into $B\left(\Gamma^{+}\left(\mathfrak{G}_{0}\right)\right)$ is a reflective covariant reducing map for the reflective covariant system $\left(N^{+},\left(N_{s, t}^{+}\right),\left(\gamma_{t}^{+}\right), \rho^{+}\right)$where

$$
\begin{aligned}
& N^{+}=B\left(\Gamma^{+}\left(\mathfrak{h}_{1}\right)\right)=B\left(\Gamma^{+}\left(\mathfrak{h}_{0}\right) \otimes \Gamma^{+}(\mathfrak{G})\right), \\
& N_{s, t}^{+}=B\left(\Gamma^{+}\left(\mathfrak{h}_{0}\right) \otimes \Gamma^{+}\left(\mathfrak{h}_{\mathfrak{j} t, s]}\right)\right) \otimes I, \\
& \gamma_{t}^{+}(T)=\Gamma^{+}\left(I \oplus S_{t}\right) T \Gamma^{+}\left(I \oplus S_{t}\right)^{-1}, \\
& \rho^{+}(T)=\Gamma^{+}(I \oplus R) T \Gamma^{+}(I \oplus R)^{-1} .
\end{aligned}
$$

When the semigroup $\left(A_{t}\right)$ in $\mathfrak{h}_{0}$ is $e^{-t}$ acting by multiplication in $\boldsymbol{C}$ the corresponding Feynman-Kac formula is that of the OrnsteinUhlenbeck velocity process, $[5,7]$, the "oscillator" formula of [12].

\section{§2. Fermion Fock Space and Second Quantisation}

Let $\mathfrak{h}$ be a Hilbert space. Let $\Gamma^{-}(\mathfrak{h})$ be the Fermion Fock space over $\mathfrak{h}$,

$$
\Gamma^{-}(\mathfrak{h})=\bigoplus_{n=0}^{\infty}\left[\bigotimes_{j=1}^{n} \mathfrak{h}\right]_{a}
$$

where the $n$-particle spaces,

$$
\mathfrak{h}_{n}=\left[\bigotimes_{j=1}^{n} \mathfrak{h}\right]_{a}, \quad n=0,1,2, \ldots
$$

are the antisymmetric parts of the $n$-fold Hilbert space tensor products of $\mathfrak{h}$ with itself, which we regard as the Hilbert space of bounded, skew symmetric $n$-fold-conjugate-linear functionals $m: \bigotimes_{j=1}^{n} \mathfrak{h}$ $\rightarrow \boldsymbol{C}$ for which

$$
\|m\|^{2}=\sum_{j_{1}, \ldots, j_{n}}\left|m\left(f_{j_{1}}, \ldots, f_{j_{n}}\right)\right|^{2}<\infty,
$$

where $\left(f_{j}\right)$ is a maximal orthonormal set in $\mathfrak{h}[9]$.

For $f \in \mathfrak{h}$ the corresponding Fock annihilation operator $a(f)$ is the bounded operator in $\Gamma(\mathfrak{G})$ whose action is

$$
a(f)\left(m_{0}, m_{1}, m_{2}, \ldots\right)=\left(m_{1}(f), 2^{\frac{1}{2}} m_{2}(f, .), 3^{\frac{1}{2}} m_{3}(f, ., .), \ldots\right) \text {. }
$$

Glearly

$$
a(f) \Omega=0, \quad f \in \mathfrak{h}
$$

where $\Omega$ or $\Omega(\mathfrak{h})$ is the Fock vacuum vector,

$$
\Omega=(1,0,0, \ldots) \text {; }
$$

also $a(f)$ depends conjugate-linearly on $f \in \mathfrak{h}$, 


$$
a(f+\lambda g)=a(f)+\bar{\lambda} a(g), \quad f, g \in \mathfrak{h}, \quad \lambda \in \boldsymbol{C}
$$

and together with its adjoint $a^{\dagger}(f)$ satisfies the canonical anticommutation relations (GAR)

$$
] a(f), a(g)[=0, \quad] a(f), a^{\dagger}(g)[=\langle f, g\rangle I, \quad f, g \in \mathfrak{h},
$$

where $] A, B[$ denotes the anticommutator

$$
] A, B[=A B+B A \text {. }
$$

It is well known that the family of operators

$$
\left\{a(f), a^{\dagger}(f): f \in \mathfrak{h}\right\}
$$

is irreducible [2] and that the set of vectors

$$
\left\{a^{\dagger}\left(f_{n}\right) \ldots a^{\dagger}\left(f_{1}\right) \Omega: n=0,1,2, \ldots, f_{1}, f_{2}, \ldots, f_{n} \in \mathfrak{h}\right\}
$$

is total in Fock space, that is the vacuum is cyclic [2]. Conversely, given a triple $(\Re, a, \Omega)$ comprising a Hilbert space $\Re$, a map $a$ from $\mathfrak{h}$ into the bounded operators on $\Re$ and a unit vector $\Omega$ in $\Omega$ such that (2.1), (2.2) and (2.3) hold and the families (2.4) and (2.5) are respectively irreducible and cyclic, then there exists a unique Hilbert space isomorphism from $\Omega$ to Fock space that maps $\Omega$ to the Fock vacuum and intertwines each $a(f), f \in \mathfrak{h}$, with the corresponding Fock annihilation operator [2].

Now let $\AA$ be a $\boldsymbol{Z}_{2}$-graded Hilbert space, that is the internal Hilbert space direct sum

$$
\Re=\Re_{0}+\Re_{1}
$$

of even and odd Hilbert sub-spaces $\Re_{0}$ and $\Omega_{1}$. The algebra $B(\Re)$ of bounded operators in $\Omega$ is $\boldsymbol{Z}_{2}$-graded [3] by the rule that $T \in B(\Re)$ is even if $T \Re_{+} \subseteq \Re_{+}$and $T \Re_{-} \subseteq \Re_{-}$, and odd if $T \Re_{+} \subseteq \Re_{-}$and $T \Re_{-} \subseteq \Re_{+}$. Equivalently, $T$ is even if $\Theta T=T \Theta$ and odd if $\Theta T=$ $-T \Theta$, where $\Theta$ is the parity operator which acts as $I$ on $\Omega_{0}$ and $-I$ on $\AA_{1}$.

If $\mathfrak{R}^{\prime}$ is a second $\boldsymbol{Z}_{2}$-graded Hilbert space then the tensor product $\mathfrak{\Omega}^{\prime \prime}=\Re \otimes \mathfrak{\Omega}^{\prime}$ is naturally $\boldsymbol{Z}_{2}$-graded by the rule

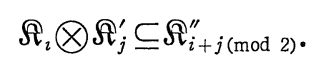

For operators $T \in B(\Re)$ and $T^{\prime} \in B\left(\Omega^{\prime}\right)$ having even and odd components $T_{0}^{\prime}, T_{1}^{\prime}$, we define an operator $T \hat{\otimes} T^{\prime}$ in $B\left(\mathscr{R} \otimes \mathscr{R}^{\prime}\right)$ by

$$
T \hat{\otimes} T^{\prime}=T \otimes T_{0}^{\prime}+T \Theta \otimes T_{1}^{\prime},
$$


where $C \otimes D$ denotes the usual Hilbert space product operator whose action on product vectors is

$$
(C \otimes D)(f \otimes g)=C f \otimes D g
$$

and $\Theta$ is the parity operator on $\AA$. Then the following rules hold:

$$
\begin{aligned}
\left(T \hat{\otimes} T^{\prime}\right)(f \otimes g) & =\mp T f \otimes T^{\prime} g \\
\left(T \hat{\otimes} T^{\prime}\right)\left(\mathrm{S} \hat{\otimes} S^{\prime}\right) & =\mp T S \hat{\otimes} T^{\prime} S^{\prime}
\end{aligned}
$$

for $S, T \in B(\Re), S^{\prime}, T^{\prime} \in B\left(\Re^{\prime}\right), f \in \Re, g \in \Re^{\prime}$ all either even or odd, where the sign is + unless, in the first case, both $T^{\prime}$ and $f$, and in the second case, both $T^{\prime}$ and $S$, are odd.

We regard the Fock space $\Gamma^{-}(\mathfrak{h})$ over $\mathfrak{h}$ as a $\boldsymbol{Z}_{2}$-graded Hilbert space, taking the even and odd subspaces to be the subspaces of vectors whose $n$-particle components vanish for $n$ odd and even respectively. Now let $\mathfrak{h}=\mathfrak{h}_{1} \oplus \mathfrak{h}_{2}$ be a direct sum. Then it is easily seen that the triple comprising the Hilbert space $\Gamma^{-}\left(\mathfrak{h}_{1}\right) \otimes \Gamma^{-}\left(\mathfrak{h}_{2}\right)$, the map

$$
\mathfrak{h} \in f=\left(f_{1}, f_{2}\right) \rightarrow a\left(f_{1}\right) \hat{\otimes} I+I \hat{\otimes} a\left(f_{2}\right)
$$

from $\mathfrak{h}$ to $B\left(\Gamma^{-}\left(\mathfrak{h}_{1}\right) \otimes \Gamma^{-}\left(\mathfrak{h}_{2}\right)\right)$ and the unit vector $\Omega\left(\mathfrak{h}_{1}\right) \otimes \Omega\left(\mathfrak{h}_{2}\right)$ satisfies the conditions (2.1), (2.2) and (2.3) together with the irreducibility and totality of the corresponding families (2.4) and (2.5). Hence there is a unique Hilbert space isomorphism, which we use to identify the two spaces, from $\Gamma^{-}\left(\mathfrak{h}_{1}\right) \otimes \Gamma^{-}\left(\mathfrak{h}_{2}\right)$ to $\Gamma^{-}(\mathfrak{G})$ mapping $\Omega\left(\mathfrak{h}_{1}\right) \otimes \Omega\left(\mathfrak{h}_{2}\right)$ to $\Omega(\mathfrak{h})$ and intertwining each $a\left(f_{1}\right) \hat{\otimes} I+I \otimes \hat{\otimes} a\left(f_{2}\right)$ with $a\left(f_{1}, f_{2}\right)$. Thus

$$
\begin{gathered}
\Gamma^{-}\left(\mathfrak{h}_{1} \oplus \mathfrak{h}_{2}\right)=\Gamma^{-}\left(\mathfrak{h}_{1}\right) \otimes \Gamma^{-}\left(\mathfrak{h}_{2}\right), \\
a\left(f_{1}, f_{2}\right)= \\
a\left(f_{1}\right) \hat{\otimes} I+I \hat{\otimes} a\left(f_{2}\right), \quad f_{j} \in \mathfrak{h}_{j}, \quad j=1,2, \\
\\
\Omega\left(\mathfrak{h}_{1} \oplus \mathfrak{h}_{2}\right)=\Omega\left(\mathfrak{h}_{1}\right) \otimes \Omega\left(\mathfrak{h}_{2}\right) .
\end{gathered}
$$

Let $C$ be a contraction from a Hilbert space $\mathfrak{h}$ to a second Hilbert space $\mathfrak{h}^{\prime}$. The second quantisation $\Gamma^{-}(C)$ of $C$ is the bounded operator from $\Gamma^{-}(\mathfrak{h})$ to $\Gamma^{-}\left(\mathfrak{h}^{\prime}\right)$ which acts on each $n$-particle space as $\bigotimes_{j=1}^{n} C$. Then the map $C \rightarrow \Gamma^{-}(C)$ is continuous for the respective strong operator topologies and satisfies the functorial rules

$$
\begin{gathered}
\Gamma^{-}(I)=I, \\
\Gamma^{-}\left(C_{1} C_{2}\right)=\Gamma^{-}\left(C_{1}\right) \Gamma^{-}\left(C_{2}\right), \\
\Gamma^{-}\left(C^{*}\right)=\Gamma^{-}\left(C^{*}\right)^{*} .
\end{gathered}
$$


Also

$$
\Gamma^{-}(C) \Omega(\mathfrak{h})=\Omega\left(\mathfrak{h}^{\prime}\right),
$$

and if $C$ is isometric, then for $f \in \mathfrak{h}$

$$
\begin{aligned}
\Gamma^{-}(G) a(f) & =a(C f) \Gamma^{-}(G), \\
\Gamma^{-}(G) a^{\dagger}(f) & =a^{\dagger}(C f) \Gamma^{-}(G) .
\end{aligned}
$$

Theorem 2.1. If $U=U_{1} \oplus U_{2}$ is a direct sum of unitary operators $U_{j}$ in $\mathfrak{h}_{j}, j=1,2$, then

$$
\Gamma^{-}(U)=\Gamma^{-}\left(U_{1}\right) \hat{\otimes} \Gamma^{-}\left(U_{2}\right) .
$$

Proof. Since the $\Gamma^{-}\left(U_{j}\right)$ are even, using (2.6) and (2.8) we have, for $f_{j} \in \mathfrak{h}_{j}, j=1,2$

$$
\begin{aligned}
& \Gamma^{-}\left(U_{1}\right) \hat{\otimes} \Gamma^{-}\left(U_{2}\right) a\left(f_{1}, f_{2}\right) \\
= & \Gamma^{-}\left(U_{1}\right) \hat{\otimes} \Gamma^{-}\left(U_{2}\right)\left[a\left(f_{1}\right) \hat{\otimes} I+I \hat{\otimes} a\left(f_{2}\right)\right] \\
= & \Gamma^{-}\left(U_{1}\right) a\left(f_{1}\right) \hat{\otimes} \Gamma^{-}\left(U_{2}\right)+\Gamma^{-}\left(U_{1}\right) \hat{\otimes} \Gamma^{-}\left(U_{2}\right) a\left(f_{2}\right) \\
= & a\left(U_{1} f_{1}\right) \Gamma^{-}\left(U_{1}\right) \hat{\otimes} \Gamma^{-}\left(U_{2}\right)+\Gamma^{-}\left(U_{1}\right) \hat{\otimes} a\left(U_{2} f_{2}\right) \Gamma^{-}\left(U_{2}\right) \\
= & {\left[a\left(U_{1} f_{1}\right) \hat{\otimes} I+I \hat{\otimes} a\left(U_{2} f_{2}\right)\right] \Gamma^{-}\left(U_{1}\right) \hat{\otimes} \Gamma^{-}\left(U_{2}\right) } \\
= & a\left(U\left(f_{1}, f_{2}\right)\right) \Gamma^{-}\left(U_{1}\right) \hat{\otimes} \Gamma^{-}\left(U_{2}\right) .
\end{aligned}
$$

Hence, using (2.14) together with the fact that $\Gamma(U)^{-1}=\Gamma\left(U^{-1}\right)$

$$
\begin{aligned}
& \Gamma^{-}(U)^{-1} \Gamma^{-}\left(U_{1}\right) \hat{\otimes} \Gamma^{-}\left(U_{2}\right) a\left(f_{1}, f_{2}\right) \\
= & \Gamma^{-}\left(U^{-1}\right) a\left(U\left(f_{1}, f_{2}\right)\right) \Gamma^{-}\left(U_{1}\right) \hat{\otimes} \Gamma^{-}\left(U_{2}\right) \\
= & a\left(f_{1}, f_{2}\right) \Gamma^{-}(U)^{-1} \Gamma^{-}\left(U_{1}\right) \hat{\otimes} \Gamma^{-}\left(U_{2}\right),
\end{aligned}
$$

that is, the operator $\Gamma^{-}(U)^{-1} \Gamma^{-}\left(U_{1}\right) \hat{\otimes} \Gamma^{-}\left(U_{2}\right)$ commutes with each $a\left(f_{1}, f_{2}\right)$. A similar argument shows that this operator commutes with each $a^{\dagger}\left(f_{1}, f_{2}\right)$, and thus with all elements of the irreducible family $\left\{a\left(f_{1}, f_{2}\right), a^{\dagger}\left(f_{1}, f_{2}\right): f_{j} \in \mathfrak{h}_{j} ; j=1,2\right\}$. Hence it is a scalar operator and for some $c \in C$

$$
\Gamma^{-}\left(U_{1}\right) \hat{\otimes} \Gamma^{-}\left(U_{2}\right)=c \Gamma^{-}(U) \text {. }
$$

Comparing the action of $\Gamma^{-}\left(U_{1}\right) \hat{\otimes} \Gamma^{-}\left(U_{2}\right)$ and $\Gamma^{-}(U)$ on the vacuum vector using (2.13) and (2.9) shows that $c=1$.

Finally we show that the second quantisation of an isometric embedding of one Hilbert space in another characterises the vacuum 
conditional expectation for the algebras on the corresponding Fock spaces. In this connection, see [4].

Theorem 2.2. Let $\mathfrak{h}_{1}$ be a direct sum, $\mathfrak{h}_{1}=\mathfrak{h}_{0} \oplus \mathfrak{h}$ and let $J$ be the isometry $\xi_{0} \rightarrow\left(\xi_{0}, 0\right)$ from $\mathfrak{h}_{0}$ into $\mathfrak{h}_{1}$. Then the map $T \rightarrow \Gamma^{-}(J) * T \Gamma^{-}(J)$ from $B\left(\Gamma^{-}\left(\mathfrak{h}_{1}\right)\right)$ to $B\left(\Gamma^{-}\left(\mathfrak{h}_{0}\right)\right)$ is the vacuum conditional expectation given $B\left(\Gamma^{-}\left(\mathfrak{h}_{0}\right)\right)$, that is, for arbitrary $T \in B\left(\Gamma^{-}\left(\mathfrak{h}_{1}\right)\right), \Gamma^{-}(J)^{*} T \Gamma^{-}(J)$ is the unique element of $B\left(\Gamma^{-}\left(\mathfrak{h}_{0}\right)\right)$ such that, for arbitrary $\phi, \chi \in \Gamma^{-}\left(\mathfrak{h}_{0}\right)$,

$$
\left\langle\psi, \Gamma^{-}(J)^{*} T \Gamma^{-}(J) \chi\right\rangle=\langle\phi \otimes \Omega(\mathfrak{h}), T \chi \otimes \Omega(\mathfrak{h})\rangle .
$$

Proof. Because of the totality of such vectors in $\Gamma^{-}\left(\mathfrak{h}_{0}\right)$ it is sufficient to establish (2.17) in the case when $\phi$ and $\chi$ are of form

$$
\phi=a^{\dagger}\left(\xi_{m}\right) \ldots a^{\dagger}\left(\xi_{1}\right) \Omega\left(\mathfrak{h}_{0}\right), \chi=a^{\dagger}\left(\eta_{n}\right) \ldots a^{\dagger}\left(\eta_{1}\right) \Omega\left(\mathfrak{h}_{0}\right)
$$

for $\xi_{1}, \ldots, \xi_{m}, \eta_{1}, \ldots, \eta_{n} \in \mathfrak{h}_{0}$. Then we have using (2.15)

$$
\begin{aligned}
\left\langle\psi, \Gamma^{-}(J) * T \Gamma^{-}(J) \chi\right\rangle \\
=\left\langle\Gamma^{-}(J) \psi, T \Gamma^{-}(J) \chi\right\rangle \\
=\left\langle\Gamma^{-}(J) a^{\dagger}\left(\xi_{m}\right) \ldots a^{\dagger}\left(\xi_{1}\right) \Omega\left(\mathfrak{h}_{0}\right), T \Gamma^{-}(J) a^{\dagger}\left(\eta_{n}\right) \ldots a^{\dagger}\left(\eta_{1}\right) \Omega\left(\mathfrak{h}_{0}\right)\right\rangle \\
=\left\langle a^{\dagger}\left(J \xi_{m}\right) \ldots a^{\dagger}\left(J \xi_{1}\right) \Gamma^{-}(J) \Omega\left(\mathfrak{h}_{0}\right), T a^{\dagger}\left(J \eta_{n}\right) \ldots a^{\dagger}\left(J \eta_{1}\right) \Gamma^{-}(J) \Omega\left(\mathfrak{h}_{0}\right)\right\rangle \\
=\left\langle a^{\dagger}\left(\xi_{m}, 0\right) \ldots a^{\dagger}\left(\xi_{1}, 0\right) \Omega\left(\mathfrak{h}_{1}\right), T a^{\dagger}\left(\eta_{n}, 0\right) \ldots a^{\dagger}\left(\eta_{1}, 0\right) \Omega\left(\mathfrak{h}_{1}\right)\right\rangle \\
=\left\langle\left(a^{\dagger}\left(\xi_{m}\right) \hat{\otimes} I\right) \ldots\left(a^{\dagger}\left(\xi_{1}\right) \hat{\otimes} I\right) \Omega\left(\mathfrak{h}_{0}\right) \otimes \Omega(\mathfrak{h}),\right. \\
\left.\quad T\left(a^{\dagger}\left(\eta_{n}\right) \hat{\otimes} I\right) \ldots\left(a^{\dagger}\left(\eta_{1}\right) \hat{\otimes} I\right) \Omega\left(\mathfrak{h}_{0}\right) \otimes \Omega(\mathfrak{h})\right\rangle \\
=\left\langle a^{\dagger}\left(\xi_{m}\right) \ldots a^{\dagger}\left(\xi_{1}\right) \Omega\left(\mathfrak{h}_{0}\right) \otimes \Omega(\mathfrak{h}), T a^{\dagger}\left(\eta_{n}\right) \ldots a^{\dagger}\left(\eta_{1}\right) \Omega\left(\mathfrak{h}_{0}\right) \otimes \Omega(\mathfrak{h})\right\rangle \\
=\langle\psi \otimes \Omega(\mathfrak{h}), T \chi \otimes \Omega(\mathfrak{h})\rangle .
\end{aligned}
$$

\section{§3. Fermion Feynman-Kac Formulae}

Let $\left(A_{t}: t \geq 0\right)$ be a strongly continuous self-adjoint contraction semigroup acting in a Hilbert space $\mathfrak{h}_{0}$, and let $\left(U_{s, t}: s \geq t\right)$ be its time-orthogonal unitary dilation in $\mathfrak{h}_{1}=\mathfrak{h}_{0} \oplus L^{2}\left(\boldsymbol{R} ; \mathfrak{h}_{0}\right)$. Let $N^{-}$be the von Neumann algebra $B\left(\Gamma^{-}\left(\mathfrak{G}_{1}\right)\right)$ and, for $s \geq t$, let $N_{s, t}^{-}$be the von Neumann algebra generated by the elements $a(\xi, f), \xi \in \mathfrak{h}_{0}, f \in \mathfrak{h}_{] t, s]}$. Let $\gamma_{t}^{-}, t \in \boldsymbol{R}$ and $\rho^{-}$be the automorphisms of $N^{-}$given by

$$
\begin{aligned}
& \gamma_{t}^{-}(T)=\Gamma^{-}\left(I \oplus S_{t}\right) T \Gamma^{-}\left(I \oplus S_{t}\right)^{-1} \\
& \rho^{-}(T)=\Gamma^{-}(I \oplus R) T \Gamma^{-}(I \oplus R)^{-1}
\end{aligned}
$$

Then $\left(N^{-},\left(N_{s, t}^{-}\right),\left(\gamma_{t}^{-}\right), \rho^{-}\right)$is a reflective covariant system. Indeed, 
it is clear that $N_{s, t}^{-} \subseteq N_{r u}^{-}$for $\left.\left.\left.] t, s\right] \subseteq\right] r, u\right]$, and that $N^{-}$is generated by the $N_{s, t}^{-}$follows from the fact that it is generated by the $a(\xi, f)$, $\xi \in \mathfrak{H}_{0}, f \in \mathfrak{h}$, that $(\xi, f) \rightarrow a(\xi, f)$ is continuous in the strong topology on $\mathfrak{h}_{1}$ and the uniform topology in $B\left(\Gamma^{-}\left(\mathfrak{h}_{1}\right)\right)$ and that $\bigcup_{s \geq t} \mathfrak{h}_{1 t, s]}$ is dense in $\mathfrak{h}$. That the inclusions (1.1) and (1.2) are satisfied follows from (2.14), (2.15) and (2.16).

Now let $j^{-}$be map from $N^{-}$to $B\left(\Gamma^{-}\left(\mathfrak{G}_{0}\right)\right)$ given by

$$
j^{-}(T)=\Gamma^{-}(J)^{*} T \Gamma^{-}(J) \text {. }
$$

By Theorem 2.2, $j^{-}$is the conditional expectation given $B\left(\Gamma^{-}\left(\mathfrak{G}_{0}\right)\right)$ in $N^{-}$.

Theorem 3.1. $j^{-}$is a reflectively covariant reducing map and $\left(\Gamma^{-}\left(U_{s, t}\right)\right.$ : $s \geq t)$ is a reflectively covariantly adapted evolution for $\left(N^{-},\left(N_{s, t}^{-}\right),\left(\gamma_{t}^{-}\right)\right.$, $\left.\rho^{-}\right)$.

Proof. The proof differs essentially from that of the corresponding result for the Boson case, Theorem 6.1 of [6], only in the argument, which will now be given, that $j$ satisfies the condition

$$
j(A B)=j(A) j(B)
$$

whenever $A \in N_{s, t}, B \in N_{r, u}$ for disjoint $\left.\left.\left.] t, s\right],\right] u, r\right]$. By our identification of $j$ as a conditional expectation and Theorem 1.1 of [6] it is sufficient to show that the corresponding von Neumann subalgebras $N_{1}$ and $N_{2}$ of $B\left(\Gamma^{-}(\mathfrak{h})\right)$ generated by the $a(f)$ for which $f$ vanishes outside $] t, s]$ and $] u, r]$, respectively, are independent in the vacuum state, that is, for arbitrary $G \in N_{1}, D \in N_{2}$

$$
\langle\Omega, C D \Omega\rangle=\langle\Omega, C \Omega\rangle\langle\Omega, D \Omega\rangle .
$$

To do this we write

$$
\Gamma^{-}(\mathfrak{h})=\Gamma^{-}\left(\mathfrak{h}_{] t, s]}\right) \Gamma^{-}\left(\mathfrak{h}_{] t, s \tau^{c}}\right)
$$

corresponding to the direct sum decomposition

$$
\mathfrak{h}=\mathfrak{h}_{] t, s]} \oplus \mathfrak{h}_{] t, s]} \text {, }
$$

and note that $N_{1}$ is generated by operators of form

$$
a\left(f_{1}\right) \hat{\otimes} I=a\left(f_{1}\right) \otimes I
$$

where $f_{1} \in \mathfrak{h}_{] t . s]}$, while $N_{2}$ is similarly generated by operators of form

$$
I \hat{\otimes} a\left(f_{2}\right)=\Theta \otimes a\left(f_{2}\right),
$$




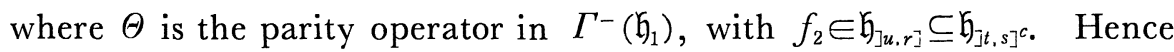
the element $C$ of $N_{1}$ is of form $C_{1} \otimes I$ for $C_{1} \in B\left(\Gamma\left(\mathfrak{h}_{] t, s]}\right)\right.$ ), while the element $D$ of $N_{2}$ is of the form $I \otimes D_{1}+\Theta \otimes D_{2}$ with $D_{1}, D_{2} \in B\left(\Gamma\left(\mathfrak{h}_{] t, s]} c\right)\right.$ ). Since $\Theta$ maps the vacuum to itself, we have, writing $\Omega_{1}=\Omega\left(\mathfrak{h}_{] t, s]}\right)$, $\Omega_{2}=\Omega\left(\mathfrak{h}_{\mathrm{J} t, s]^{c}}\right)$

$$
\begin{aligned}
\langle\Omega, & C D \Omega\rangle \\
& =\left\langle\Omega_{1} \otimes \Omega_{2}, \quad\left(C_{1} \otimes I\right)\left(I \otimes D_{1}+\Theta \otimes D_{2}\right) \Omega_{1} \otimes \Omega_{2}\right\rangle \\
& =\left\langle\Omega_{1} \otimes \Omega_{2}, C_{1} \otimes\left(D_{1}+D_{2}\right) \Omega_{1} \otimes \Omega_{2}\right\rangle \\
& =\left\langle\Omega_{1}, C_{1} \Omega_{1}\right\rangle\left\langle\Omega_{2}, \quad\left(D_{1}+D_{2}\right) \Omega_{2}\right\rangle .
\end{aligned}
$$

Setting $C$ and $D$, respectively, equal to the identity shows that

$$
\begin{aligned}
& \langle\Omega, D \Omega\rangle=\left\langle\Omega_{2}, \quad\left(D_{1}+D_{2}\right) \Omega_{2}\right\rangle, \\
& \langle\Omega, C \Omega\rangle=\left\langle\Omega_{1}, C_{1} \Omega_{1}\right\rangle .
\end{aligned}
$$

Hence in general we obtain

$$
\langle\Omega, C D \Omega\rangle=\langle\Omega, C \Omega\rangle\langle\Omega, D \Omega\rangle
$$

as required.

As in the Boson case, the reduced evolution corresponding to the reducing map $j^{-}$of the evolution $\Gamma^{-}\left(U_{s, t}\right)$ is the second quantisation of $\left(A_{t}\right)$ :

$$
\begin{aligned}
j^{-}\left(\Gamma^{-}\left(U_{s, t}\right)\right) & =\Gamma^{-}(J) * \Gamma^{-}\left(U_{s, t}\right) \Gamma^{-}(J) \\
& =\Gamma^{-}\left(J^{*} U_{s, t} J\right) \\
& =\Gamma^{-}\left(A_{s-t}\right) .
\end{aligned}
$$

In the case when $A_{t}=e^{-t}$ acting by multiplication in $\boldsymbol{C}$, this is the semigroup $I \oplus e^{-t}$ in $\Gamma^{-}(\boldsymbol{C})=\boldsymbol{C} \oplus \boldsymbol{C}$; its infinitesimal generator is thus the two-level Hamiltonian with eigenvalues 0 and 1. Perturbations $j^{-}(B)$ for which the $\Gamma^{-}\left(U_{t, s}\right) B \Gamma^{-}\left(U_{t, s}\right)^{-1}$ all commute, so that the Feynman-Kac cocycle takes exponential-integral form, must themselves be diagonal so that, at least in the case of time-independent perturbations, the Feynman-Kac formula which results seems unlikely to be of value for computations. It bears the same relationship to the Fermion Ornstein-Uhlenbeck velocity process

$$
a(t)=e^{-t} a_{0} \hat{\otimes} I+\sqrt{2} I \hat{\otimes} \int_{0}^{t} e^{-(t-r)} d A_{r}, \quad t \geq 0,
$$

where $\left(A_{t}\right)$ is the Fermion Wiener (Brownian motion) process

$$
A_{t}=a\left(\chi_{[0, t]}\right), \quad t \geq 0
$$


as does the oscillator process formula of the Boson case to the Boson canonical Ornstein-Uhlenbeck velocity process of $[6,7]$.

\section{§4. Extremal Universally Invariant Second Quantisation}

Let $\mathfrak{h}$ be a Hilbert space and let $\Gamma^{+}(\mathfrak{h})$ be its Boson Fock space, with vacuum vector $\Omega^{+}$or $\Omega^{+}(\mathfrak{h})$. Denote by $\mathbb{V}(f), f \in \mathfrak{h}$ the Weyl operators over $\mathfrak{h}$; these form an irreducible family of unitary operators in $\Gamma^{+}(\mathfrak{h})$ satisfying

$$
\begin{gathered}
W(f) W(g)=\exp \left\{-\frac{1}{2} i \operatorname{Im}\langle f, g\rangle\right\} W(f+g), \\
\left\langle\Omega^{+}, W(f) \Omega^{+}\right\rangle=\exp \left\{-\frac{1}{4} \mid\|f\|^{2}\right\},
\end{gathered}
$$

moreover the map $f \rightarrow W(f)$ is strongly continuous on $\mathfrak{h}$.

Now let $\overline{\mathfrak{h}}$ be the dual Hilbert space of $\mathfrak{h}$ and for $f \in \mathfrak{h}$ let $\bar{f}$ denote the element $g \rightarrow\langle f, g\rangle$ of $\overline{\mathfrak{h}}$. Let $K$ be the natural conjugate isomorphism $f \rightarrow \bar{f}$ from $\mathfrak{h}$ to $\overline{\mathfrak{h}}$ and for $T \in B(\mathfrak{G})$ let $\bar{T}$ denote the element $K T K^{-1}$ of $B(\overline{\mathfrak{G}})$. By identifying the dual of a direct sum with the direct sum of the duals through the isomorphism

$$
\left(f_{1}, f_{2}, \ldots\right) \rightarrow\left(\bar{f}_{1}, \bar{f}_{2}, \ldots\right)
$$

and the dual of a tensor product with the tensor product of the duals through the isomorphism which extends the map

$$
\left(f_{1} \otimes f_{2} \otimes \ldots\right)^{-} \rightarrow \bar{f}_{1} \otimes \bar{f}_{2} \otimes \ldots,
$$

we identify the dual of the Fock space $\Gamma^{+}(\mathfrak{G})$ with the Fock space $\Gamma^{+}(\overline{\mathfrak{h}})$ of the dual, in such a way that for any contraction $C$, the second quantisations $\Gamma^{+}(G)$ and $\Gamma^{+}(\bar{G})$ in $\Gamma^{+}(\mathfrak{G})$ and $\Gamma^{+}(\overline{\mathfrak{G}})=\overline{\Gamma^{+}(\mathfrak{h})}$ satisfy

$$
\overline{\Gamma^{+}(C)}=\Gamma^{+}(\bar{C}) .
$$

Now set

$$
\begin{gathered}
\Gamma(\mathfrak{h})=\Gamma^{+}(\mathfrak{h}) \otimes \Gamma^{+}(\overline{\mathfrak{h}})=\Gamma^{+}(\mathfrak{h} \oplus \overline{\mathfrak{h}}), \\
\Omega=\Omega(\mathfrak{h})=\Omega^{+}(\mathfrak{h}) \otimes \Omega^{+}(\overline{\mathfrak{h}})=\Omega^{+}(\mathfrak{h} \oplus \overline{\mathfrak{h}}) .
\end{gathered}
$$

If $\mathfrak{h}=\bigoplus_{j=1}^{n} \mathfrak{h}_{j}$ is a finite direct sum then by combining the corresponding isomorphism for the Fock case with the natural permutation isomorphism from 


$$
\Gamma(\mathfrak{h})=\Gamma^{+}(\mathfrak{h}) \otimes \Gamma^{+}(\overline{\mathfrak{h}})=\left\{\bigotimes_{j=1}^{n} \Gamma^{+}\left(\mathfrak{h}_{j}\right)\right\} \otimes\left\{\bigotimes_{j=1}^{n} \Gamma^{+}\left(\overline{\mathfrak{h}}_{j}\right)\right\}
$$

to

$$
\bigotimes_{j=1}^{n}\left\{\Gamma^{+}\left(\mathfrak{h}_{j}\right) \otimes \Gamma^{+}\left(\overline{\mathfrak{h}}_{j}\right)\right\}=\bigotimes_{j=1}^{n} \Gamma\left(\mathfrak{h}_{j}\right),
$$

we obtain an isomorphism from $\Gamma(\mathfrak{h})$ to $\bigotimes_{j=1}^{n} \Gamma\left(\mathfrak{h}_{j}\right)$ which we use to identify these spaces. Note that in this identification

$$
\Omega\left(\bigoplus_{j=1}^{n} \mathfrak{h}_{j}\right)=\bigotimes_{j=1}^{n} \Omega\left(\mathfrak{h}_{j}\right) .
$$

If $C$ is a contraction from $\mathfrak{h}$ to a Hilbert space $\mathfrak{h}^{\prime}$, we write

$$
\Gamma(C)=\Gamma^{+}(G) \otimes \Gamma^{+}(\bar{C}) .
$$

Because of corresponding properties of the Fock second quantisations, $\Gamma(C)$ is a contraction, $C \rightarrow \Gamma(C)$ is strongly continuous and the functorial rules

$$
\begin{gathered}
\Gamma(I)=I, \\
\Gamma\left(C_{1} C_{2}\right)=\Gamma\left(C_{1}\right) \Gamma\left(C_{2}\right), \\
\Gamma\left(C^{*}\right)=\Gamma\left(C_{1}\right)^{*}
\end{gathered}
$$

are satisfied ; moreover if $C=\bigoplus_{j=1}^{n} C_{j}$ is a finite direct sum then

$$
\Gamma(G)=\bigotimes_{j=1}^{n} \Gamma\left(C_{j}\right) \text {. }
$$

Now let $\sigma$ be a real number $>1$, fixed once and for all. Let $\alpha$ be the positive number such that

$$
\sigma^{2}=\cosh 2 \alpha .
$$

Then it is easily verified that the family of unitary operators $W_{\sigma}=$ $\left(W_{\sigma}(f): f \in \mathfrak{h}\right)$ defined by

$$
W_{\sigma}(f)=W(\cosh \alpha f) \otimes W(\sinh \alpha \bar{f})
$$

forms a RCGR over $\mathfrak{h}$ (that is, is strongly continuous on finitedimensional subspaces of $\mathfrak{h}$ and satisfies

$$
\left.W_{\sigma}(f) W_{\sigma}(g)=\exp \left\{-\frac{1}{2} i \operatorname{Im}\langle f, g\rangle\right\} W_{\sigma}(f+g), \quad f, g \in \mathfrak{h}\right) .
$$

Moreover

$$
\left\langle\Omega, W_{\sigma}(f) \Omega\right\rangle=\exp \left\{-\frac{1}{4} \sigma^{2}\|f\|^{2}\right\} .
$$

$W_{\sigma}$ is thus a realisation of the RCGR corresponding to an extremal 
universally invariant state of the CCR algebra in the sense of [10]. Similarly, if

$$
W_{\sigma}^{\prime}(\bar{f})=W(\sinh \alpha f) \otimes W(\cosh \alpha \bar{f}), \quad \bar{f} \in \overline{\mathfrak{h}}
$$

then $W^{\prime}$ is an RCGR over $\dot{\mathfrak{h}}$ such that

$$
\left\langle\Omega, W_{\sigma}^{\prime}(\bar{f}) \Omega\right\rangle=\exp \left\{-\frac{1}{4} \sigma^{2}\|\bar{f}\|^{2}\right\} .
$$

It can be verified directly that every element $W_{\sigma}(f)$ commutes with every $W_{\sigma}^{\prime}(\bar{g})$. Moreover, since

$$
\begin{aligned}
W_{\sigma}(f) W_{\sigma}^{\prime}(\bar{g}) & =W(\cosh \alpha f+\sinh \alpha g) \otimes W(\sinh \alpha \bar{f}+\cosh \alpha \bar{g}) \\
& =W(\cosh \alpha f+\sinh \alpha g, \sinh \alpha \bar{f}+\cosh \alpha \bar{g}),
\end{aligned}
$$

and the real linear map in $\mathfrak{h} \oplus \overline{\mathfrak{h}}$

$$
T_{\alpha}:(f, \bar{g}) \rightarrow(\cosh \alpha f+\sinh \alpha g, \sinh \alpha \bar{f}+\cosh \alpha \bar{g})
$$

is clearly invertible, we see that the operators $W_{\sigma}(f), W_{\sigma}^{\prime}(\bar{g}), f, g \in \mathfrak{h}$, jointly generate the elements of the Fock RCCR over $\mathfrak{h} \oplus \overline{\mathfrak{h}}$. Since this is irreducible it follows that the von Neumann algebras generated by the representations $W_{\sigma}$ and $W_{\sigma}^{\prime}$ are mutually commuting factors (in fact they are each other's commutants); we denote them by $N_{\circ}$ and $N_{\sigma}^{\prime}$ or $N_{\sigma}(\mathfrak{h})$ and $N_{\sigma}^{\prime}(\mathfrak{h})$ respectively. $\Omega$ is cyclic and separating for both $N_{\sigma}$ and $N_{\sigma}^{\prime}$.

Let $U$ be a unitary operator in $\mathfrak{h}$. From the corresponding property for the Fock case we see that, for $f \in \mathfrak{h}$,

$$
\begin{aligned}
& \Gamma(U) W_{\sigma}(f) \Gamma(U)^{-1}=W_{\sigma}(U f), \\
& \Gamma(U) W_{\sigma}^{\prime}(\bar{f}) \Gamma(U)^{-1}=W_{\sigma}^{\prime}(\bar{U} \bar{f}),
\end{aligned}
$$

thus conjugation by $\Gamma(U)$ induces automorphisms of $N_{\sigma}$ and $N_{\sigma}^{\prime}$. It is important for us to know when these automorphisms are inner, or equivalently when $\Gamma(U)$ can be factorized in the form

$$
\Gamma(U)=\Lambda(U) \Lambda^{\prime}(U)
$$

with $\Lambda(U)$ and $\Lambda^{\prime}(U)$ unitary elements of $N_{\sigma}$ and $N_{\sigma}^{\prime}$ respectively. Because $N_{\sigma}$ and $N_{\sigma}^{\prime}$ are factors it is clear that, if it exists, such a factorization is non-unique only to the extent that $\Lambda(U)$ and $\Lambda^{\prime}(U)$ may be replaced by $\alpha \Lambda(U)$ and $\alpha^{-1} \Lambda^{\prime}(U)$ respectively, where $\alpha$ is an element of the group $\boldsymbol{T}$ of complex numbers of unit modulus. It is also clear that the set of unitary operators for which the factorization (4.11) exists is a group under multiplication, of which the map $U \rightarrow$ 
$\boldsymbol{T} \Lambda(U)$ is a unitary ray representation [1]. We recall from [1] that a unitary ray representation $G \ni g \rightarrow \boldsymbol{T} U_{g}$ of a topological group $G$ is said to be continuous if, for an arbitrary vector $\psi$ there is a neighbourhood $\mathfrak{R}$ of the neutral element of $G$ such that whenever $g h^{-1} \in \mathfrak{N}$

$$
\inf _{\alpha, \beta \in T}\left\|\alpha U_{g} \psi-\beta U_{h} \psi\right\|<\varepsilon
$$

Theorem 4.1. The group $\mathscr{U}_{2}(\mathfrak{h})$ of unitary operators in $\mathfrak{h}$ for which the factorisation (4.11) exists consists precisely of those unitary operators $U$ for which $U-I$ is a Hilbert-Schmidt operator. Moreover if $\mathscr{U}_{2}(\mathfrak{h})$ is topologised by the metric

$$
\rho\left(U_{1}, U_{2}\right)=\left\|U_{1}-U_{2}\right\|_{2},
$$

where \|\|$_{2}$ is the Hilbert-Schmidt norm, then the map $U \rightarrow \boldsymbol{T} \Lambda(U)$ is a continuous unitary ray representation of $\mathscr{U}_{2}(\mathfrak{h})$.

Proof. Inverting the relation (4.8), we have that for all $f, g \in \mathfrak{h}$,

$$
W(f, \bar{g})=W_{o}(\cosh \alpha f-\sinh \alpha g) W_{\sigma}^{\prime}(-\sinh \alpha \bar{f}+\cosh \alpha \bar{g})
$$

where $W($,$) is the Fock RCGR over \mathfrak{h} \oplus \overline{\mathfrak{h}}$. Suppose that $\Gamma(U)$ admits the factorisation (4.11). Then since $\Lambda(U)$ commutes with each $W_{\sigma}^{\prime}(\bar{k}), \bar{k} \in \overline{\mathfrak{h}}$ and $\Lambda^{\prime}(U)$ with each $W_{\sigma}(h), h \in \mathfrak{h}$ we have

$$
\begin{aligned}
\Lambda(U) & W(f, \bar{g}) \Lambda(U)^{-1} \\
& =\Lambda(U) W_{o}(\cosh \alpha f-\sinh \alpha g) W_{\sigma}^{\prime}(-\sinh \alpha \bar{f}+\cosh \alpha \bar{g}) \Lambda(U)^{-1} \\
& =\Lambda(U) W_{\sigma}(\cosh \alpha f-\sinh \alpha g) \Lambda(U)^{-1} W_{\sigma}^{\prime}(-\sinh \alpha \bar{f}+\cosh \alpha \bar{g}) \\
& =\Gamma(U) W_{\sigma}(\cosh \alpha f-\sinh \alpha g) \Gamma(U)^{-1} W_{\sigma}^{\prime}(-\sinh \alpha \bar{f}+\cosh \alpha \bar{g}) \\
& =W_{\sigma}(\cosh \alpha U f-\sinh \alpha U g) W_{\sigma}^{\prime}(-\sinh \alpha \bar{f}+\cosh \alpha \bar{g})
\end{aligned}
$$

using (4.9). By (4.12) we can write this as

$$
\Lambda(U) W(f, \bar{g}) \Lambda(U)^{-1}=W\left(f^{\prime}, \tilde{g}^{\prime}\right)
$$

where

$$
\begin{aligned}
& f^{\prime}=\left(\cosh ^{2} \alpha U-\sinh ^{2} \alpha I\right) f+(I-U) \sinh \alpha \cosh \alpha g, \\
& g^{\prime}=(U-I) \sinh \alpha \cosh \alpha f+\left(\cosh ^{2} \alpha I-\sinh ^{2} \alpha U\right) g .
\end{aligned}
$$

Suppose, conversely, that there exists a unitary operator $\Lambda(U)$ in $\Gamma$ (h) such that, for all $f, g \in \mathfrak{h}$,

$$
\Lambda(U) W(f, \bar{g}) \Lambda(U)^{-1}=W\left(f^{\prime}, \bar{g}^{\prime}\right)
$$


with $f^{\prime}, g^{\prime}$ given by (4.13), (4.14). Replacing $f$ and $g$ by $\cosh \alpha f+$ $\sinh \alpha g$ and $\sinh \alpha f+\cosh \alpha g$ in (4.15) we have that, for arbitrary $f, g \in \mathfrak{h}$,

$$
\begin{aligned}
& \Lambda(U) W(\cosh \alpha f+\sinh \alpha g, \sinh \alpha \bar{f}+\cosh \alpha \bar{g}) \Lambda(U)^{-1} \\
& =W(\cosh \alpha U f+\sinh \alpha g, \sinh \alpha U \bar{f}+\cosh \alpha \bar{g}),
\end{aligned}
$$

that is, using (4.12), that

$$
\Lambda(U) W_{\sigma}(f) W_{\sigma}^{\prime}(\bar{g}) \Lambda(U)^{-1}=W_{\sigma}(U f) W_{\sigma}^{\prime}(\bar{g}) .
$$

Setting $f=0$ shows that $\Lambda(U)$ commutes with each $W_{\sigma}^{\prime}(g)$ and hence belongs to $N_{\sigma}$. Setting $g=0$ shows that, for all $f \in \mathfrak{h}$

$$
\begin{aligned}
\Lambda(U) W_{\sigma}(f) \Lambda(U)^{-1} & =W_{\sigma}(U f) \\
& =\Gamma(U) W_{\sigma}(f) \Gamma(U)^{-1}
\end{aligned}
$$

and hence that if

$$
\Lambda^{\prime}(U)=\Lambda(U)^{-1} \Gamma(U)
$$

then $\Lambda^{\prime}(U)$ commutes with all $W_{\sigma}(f)$ and so belongs to $N_{\sigma}^{\prime}$. Thus

$$
\Gamma(U)=\Lambda(U) \Lambda^{\prime}(U)
$$

admits a factorisation of type (4.11).

We have thus shown that $\Gamma(U)$ admits a factorisation of type (4.11) if and only if there exists a unitary operator (which can be taken to be $\Lambda(U)$ ) such that, for arbitrary $f, g \in \mathfrak{h}$,

$$
\Lambda(U) W(f, \bar{g}) \Lambda(U)^{-1}=W\left(f^{\prime}, \bar{g}^{\prime}\right),
$$

where $f^{\prime}, g^{\prime}$ are given by (4.13), (4.14). But according to Shale's theorem [11] on the unitary implementability of linear canonical transformations in the Fock RCCR, such a unitary operator $\Lambda(U)$ exists if and only if the real-linear map $L:(f, \bar{g}) \rightarrow\left(f^{\prime}, \bar{g}^{\prime}\right)$ in the real Hilbert space got by equipping $\mathfrak{h} \oplus \overline{\mathfrak{h}}$ with the real part of its original inner product has the property that $\left(L^{*} L\right)^{\frac{1}{2}}-I$ is HilbertSchmidt, or equivalently that

$$
L^{*} L-I=\left(\left(L^{*} L\right)^{\frac{1}{2}}+I\right)\left(\left(L^{*} L\right)^{\frac{1}{2}}-I\right)
$$

is Hilbert-Schmidt. Hence to complete the proof of the first statement of the theorem we need to show that $L^{*} L-I$ is Hilbert-Schmidt in the real Hilbert space $\mathfrak{h} \oplus \overline{\mathfrak{h}}$ if and only if $U-I$ is Hilbert-Schmidt in $\mathfrak{h}$.

To do this we represent the operator $L$ in $\mathfrak{h} \oplus \overline{\mathfrak{h}}$ in matrix form as 


$$
\begin{aligned}
& {\left[\begin{array}{lc}
\cosh ^{2} \alpha U-\sinh ^{2} \alpha I & \cosh \alpha \sinh \alpha(I-U) K^{-1} \\
\cosh \alpha \sinh \alpha K(\bar{U}-\bar{I}) & \cosh ^{2} \alpha \bar{I}-\sinh ^{2} \alpha \bar{U}
\end{array}\right] } \\
= & {\left[\begin{array}{lc}
I+\cosh ^{2} \alpha D & -\cosh \alpha \sinh ^{2} \alpha K^{-1} \\
\cosh \alpha \sinh \alpha K \bar{D} & \bar{I}-\sinh ^{2} \alpha \bar{D}
\end{array}\right] }
\end{aligned}
$$

where $D=U-I$. After some manipulation using the unitarity condition $U^{*} U=I$ for $U$ in the form

$$
D+D^{*}+D^{*} D=0
$$

we find that $L^{*} L-I$ is represented by the matrix

$$
2 \cosh \alpha \sinh \alpha\left[\begin{array}{cc}
-\cosh \alpha \sinh \alpha\left(D+D^{*}\right) & \left(\sinh ^{2} \alpha D+\cosh ^{2} \alpha D^{*}\right) K^{-1} \\
K\left(\cosh \alpha \bar{D}+\sinh ^{2} \alpha \bar{D}^{*}\right) & -\cosh \alpha \sinh \alpha\left(\bar{D}+\bar{D}^{*}\right)
\end{array}\right]
$$

and is thus Hilbert-Schmidt if and only if $D=U-I$ is HilbertSchmidt as required.

From the form (4.16) of the matrix of $L$ it is clear that the map $U \rightarrow L$ is continuous from the topological group $\mathscr{U}_{2}(\mathfrak{h})$ into the group $\mathscr{L}_{2}(\mathfrak{h} \oplus \overline{\mathfrak{h}})$ of real-linear transformations in $\mathfrak{h} \oplus \overline{\mathfrak{h}}$ differing by Hilbert-Schmidt operators from the identity, topologised in the same way as $\mathscr{U}_{2}(\mathfrak{G})$. Now in [11] it is proved that the unitary operators implementing unitarily implementable linear-canonical transformations constitute a continuous unitary-ray representation of the subgroup of $\mathscr{L}_{2}(\mathfrak{h} \oplus \overline{\mathfrak{h}})$ consisting of such linear canonical transformations. Combining this fact with the continuity of the map $U \rightarrow L$ shows that $U \rightarrow \boldsymbol{T} \Lambda(U)$ is a continuous unitary ray representation of $\mathscr{U}_{2}(\mathfrak{G})$ as claimed.

\section{§5. Time-Orthogonal Unitary Dilations of Semigroups with Trace-Class Infinitesimal Generator}

Let $\left(U_{s, t}\right)$ acting in $\mathfrak{h}_{1}=\mathfrak{h}_{0} \oplus \mathfrak{h}$, where $\mathfrak{h}=L^{2}\left(\boldsymbol{R} ; \mathfrak{h}_{0}\right)$, be the timeorthogonal unitary dilation of the strongly continuous self-adjoint contraction semigroup acting in $\mathfrak{h}_{0}$

$$
A_{t}=e^{-t H}
$$

with infinitesimal generator $H$.

Theorem 5.1. A necessary and sufficient condition that the elements $U_{s, t}$ belong to the group $\mathscr{U}_{2}\left(\mathfrak{h}_{1}\right)$ is that the infinitesimal generator $H$ be 
trace-class. If this is the case then the evolution $\left(U_{s, t}\right)$ is continuous in the sense of the topology of $\mathscr{U}_{2}\left(\mathfrak{h}_{1}\right)$.

Proof. As in [6], we write $U_{s, t}$ in matrix form,

$$
U_{s, t}=\left[\begin{array}{ll}
A_{s, t} & B_{s, t} \\
C_{s, t} & I+D_{s, t}
\end{array}\right]
$$

where $A_{s, t}=A_{s-t}$ and the actions of $B_{s, t}: \mathfrak{h} \rightarrow \mathfrak{h}_{0}, C_{s, t}: \mathfrak{h}_{0} \rightarrow \mathfrak{h}$ and $D_{s, t}$ : $\mathfrak{h} \rightarrow \mathfrak{h}$ are respectively

$$
\begin{gathered}
B_{s, t} f=-(2 H)^{\frac{1}{2}} \int \chi_{] t, s]}(x) A_{s-x} f(x) d x, \\
\left(C_{s, t} \zeta\right)(x)=\chi_{] t, s]}(x) A_{x-t}(2 H)^{\frac{1}{2} \zeta}, \\
\left(D_{s, t} f\right)(x)=-2 \int \chi_{\lceil t, s]}(x) \chi_{] y, s]}(x) H A_{x-y} f(y) d y .
\end{gathered}
$$

If each $U_{s, t}$ differs from the identity by a Hilbert-Schmidt operator then clearly $A_{s-t}=J^{*} U_{s, t} J$ has the same property and thus, in particular, has a discrete spectral resolution. Hence we may assume without loss of generality that $H$ has a discrete spectral resolution, hence that there exists a maximal orthonormal set $\left(\zeta_{j}\right)$ of eigenvectors of $H$ in terms of which $H$ can be expanded as a strongly convergent sum

$$
H=\sum \lambda_{j} \zeta_{j} \otimes \bar{\zeta}_{j},
$$

where $f \otimes \bar{g}$ is the rank one operator $\phi \rightarrow\langle g, \phi\rangle f$. The matrix elements $A_{s, t}-I, B_{s, t}, C_{s, t}, D_{s, t}$ of $U_{s, t}-I$ are then given by

$$
\begin{gathered}
A_{s, t}-I=\sum_{j}\left(e^{-\lambda_{j}(s-t)}-I\right) \zeta_{j} \otimes \bar{\zeta}_{j}, \\
B_{s, t}=\sum_{j} \zeta_{j} \otimes \bar{f}_{s, t}^{j}, \quad C_{s, t}=\sum_{j} g_{s, t}^{j} \otimes \bar{\zeta}_{j},
\end{gathered}
$$

where

$$
\begin{aligned}
& f_{s, t}^{j}(x)=-\chi_{] t, s]}(x) \sqrt{2 \lambda_{j}} e^{-\lambda_{j}(s-x)} \zeta_{j}, \\
& g_{s, t}^{j}(x)=\chi_{\exists t, s]}(x) e^{-\lambda_{j}(x-t)} \sqrt{2 \lambda_{j}} \zeta_{j},
\end{aligned}
$$

and

$$
D_{s, t}=\sum_{j} D_{s, t}^{j}
$$

where

$$
D_{s, t}^{j} f(x)=-2 \int \chi_{] t, s]}(y) \chi_{\sqsupset y, s]}(x) \lambda_{j} e^{-\lambda_{j}(x-y)}\left\langle\zeta_{j}, f(y)\right\rangle \zeta_{j} d y .
$$

Identifying the spaces $L^{2}\left(\boldsymbol{R} ; \mathfrak{h}_{0}\right)$ and $L^{2}(\boldsymbol{R}) \otimes \mathfrak{h}_{0}$ by means of the 
isomorphism that extends the map $f \zeta \rightarrow f \otimes \zeta$ we see that $D_{s, t}^{j}$ can be written

$$
D_{s, t}^{j}=d_{s, t}^{j} \otimes\left(\zeta_{j} \otimes \bar{\zeta}_{j}\right)
$$

where $d_{s, t}^{j}$ is the integral operator in $L^{2}(\boldsymbol{R})$

$$
d_{s, t}^{j} f(x)=-2 \int \chi_{j t, s]}(y) \chi_{j y, s]}(x) \lambda_{j} e^{-\lambda_{j}(x-y)} f(y) d y .
$$

Since the ranges of $\zeta_{j} \otimes \bar{f}_{s, t}^{j},\left(g_{s, t}^{j} \otimes \bar{\zeta}_{j}\right) *$ and $D_{s, t}^{j}$ are mutually orthogonal for different $j$, the finite or infinite Hilbert-Schmidt norms of $B_{s, t}, C_{s, t}$ and $D_{s, t}$ are given by

$$
\begin{aligned}
\left\|B_{s, t}\right\|_{2}^{2} & =\sum_{j}\left\|\zeta_{j} \otimes \bar{f}_{s, t}^{j}\right\|^{2}=\sum_{j} \| f_{s, t}^{j}||^{2}=\sum_{j}\left(1-e^{-2 \lambda_{j}(s-t)}\right) \\
\left\|C_{s, t}\right\|_{2}^{2} & =\sum_{j}\left\|g_{s, t}^{j} \otimes \bar{\zeta}_{j}\right\|^{2}=\sum_{j}\left\|g_{s, t}^{j}\right\|^{2}=\sum_{j}\left(1-e^{-2 \lambda_{j}(s-t)}\right) \\
\left\|D_{s, t}\right\|_{2}^{2} & =\sum_{j}\left\|D_{s, t}^{j}\right\|_{2}^{2}=\sum_{j}\left\|d_{s, t}^{j}\right\|_{2}^{2} \\
& =4 \sum_{j} \iint_{\mid}\left|\chi_{] t, s]}(y) \chi_{J y, s]}(x) \lambda_{j} e^{-\lambda_{j}(x-y)}\right|^{2} d x d y \\
& =4 \sum_{j} \lambda_{j}^{2} \int_{t}^{s} \int_{y}^{s} e^{-2 \lambda_{j}(x-y)} d x d y \\
& =\sum_{j}\left(1-e^{-2 \lambda_{j}(s-t)}\right)+2 \sum_{j} \lambda_{j}(s-t) .
\end{aligned}
$$

Also from (3.5)

$$
\left\|A_{s, t}-I\right\|_{2}^{2}=\sum_{j}\left(e^{-\lambda_{j}(s-t)}-1\right)^{2} .
$$

Hence $A_{s, t}-I, B_{s, t}, C_{s, t}$ and $D_{s, t}$, and hence also $U_{s, t}-I$, have finite Hilbert-Schmidt norm if and only if each of the series

$$
\sum_{j}\left(e^{-\lambda_{j}(s-t)}-1\right)^{2}, \quad \sum_{j}\left(1-e^{-2 \lambda_{j}(s-t)}\right), \quad \sum_{j} \lambda_{j}(s-t)
$$

converges. But convergence of the last of these implies that of the other two. Hence $U_{s, t}-I$ is Hilbert-Schmidt for all $s \geq t$ if and only if $\sum_{j} \lambda_{j}$ converges, that is $H$ is trace-class.

If this is the case then, for $r>s \geq t$ from (5.1),

$$
\mid A_{r, t}-A_{s, t} \|_{2}^{2}=\sum_{j}\left(e^{-\lambda_{j}(r-t)}-e^{-\lambda_{j}(s-t)}\right) \rightarrow 0
$$

as $r \rightarrow s$, by the monotone convergence theorem, from (5.2)

$$
\begin{aligned}
\left\|B_{r, t}-B_{s, t}\right\|_{2}^{2} & =\sum_{j}\left\|f_{r, t}^{j}-f_{s, t}^{j}\right\|^{2} \\
& =\sum_{j} 2 \lambda_{j}\left\{\int_{t}^{s}\left(e^{-\lambda_{j}(r-x)}-e^{-\lambda_{j}(s-x)}\right)^{2} d x+\int_{s}^{r} e^{-2 \lambda_{j}(r-x)} d x\right\}
\end{aligned}
$$




$$
\begin{aligned}
&= \sum_{j}\left\{\left(e^{-\lambda_{j} r}-e^{-\lambda_{j} s}\right)^{2}\left(e^{2 \lambda_{j} s}-e^{2 \lambda_{j} t}\right)+1-e^{-2(r-s) \lambda_{j}}\right\} \\
& \leq e^{2 s \max \lambda_{j}} \sum_{j}\left(e^{-\lambda_{j} r}-e^{-\lambda_{j} s}\right)^{2}+2(r-s) \sum_{j} \lambda_{j} \\
& \rightarrow 0 \text { as } r \rightarrow s, \\
&\left\|G_{r, t}-C_{s, t}\right\|_{2}^{2}=\sum_{j}\left\|g_{r, t}^{j}-g_{s, t}^{j}\right\|^{2} \\
&=2 \sum_{j} \lambda_{j} \int_{s}^{r} e^{-2 \lambda_{j}(x-t)} d x \\
&=\sum_{j}\left(e^{-2 \lambda_{j}(s-t)}-e^{-2 \lambda_{j}(r-t)}\right) \\
& \rightarrow 0 \text { as } r \rightarrow s,
\end{aligned}
$$

while from (5.3), (5.4)

$$
\begin{aligned}
\left\|D_{r, t}-D_{s, t}\right\|_{2}^{2} & =\sum_{j}\left\|d_{r, t}^{j}-d_{s, t}^{j}\right\|_{\mid}^{2} \\
& =4 \sum_{j} \lambda_{j}^{2}\left\{\int_{s}^{r} \int_{t}^{s} e^{-2 \lambda_{j}(x-y)} d x d y+\int_{s}^{r} \int_{y}^{r} e^{-2 \lambda_{j}(x-y)} d x d y\right\} \\
& \leq 4 \sum_{j} \lambda_{j}^{2}\left\{(r-s)(s-t)+(r-s)^{2}\right\} \\
& \rightarrow 0 \text { as } r \rightarrow s .
\end{aligned}
$$

Hence $U_{s, t}$ is continuous in $s$ for fixed $t$. A similar argument shows that $U_{s, t}$ is continuous in $t$ for fixed $s$.

\section{§6. Factorisation of $\left(\Gamma\left(U_{s, t}\right)\right)$}

Let $\left(A_{t}: t \geq 0\right)$ be a strongly continuous self-adjoint contraction semigroup in the Hilbert space $\mathfrak{h}$ having trace-class infinitesimal generator $H$. Let $\left(U_{s, t}: s \geq t\right)$ be the time-orthogonal unitary dilation of $\left(A_{t}\right)$. Combining Theorems 4.1 and 5.1, we have that, for arbitrary $s \geq t, \Gamma\left(U_{s, t}\right)$ admits a factorisation

$$
\Gamma\left(U_{s, t}\right)=\Lambda_{s, t} \Lambda_{s, t}^{\prime}
$$

into unitary operators $\Lambda_{s, t}$ in $N_{\sigma}$ and $\Lambda_{s, t}^{\prime}$ in $N_{\sigma}^{\prime}$ which is non-unique only to the extent of multiplying $\Lambda_{s, t}$ by an arbitrary element of $\boldsymbol{T}$ and $\Lambda_{s, t}^{\prime}$ by its reciprocal. Moreover the unitary ray-valued map $(s, t) \rightarrow \boldsymbol{T} \Lambda_{s, t}=\boldsymbol{\Lambda}_{s, t}$ is separately continuous in its two arguments. It follows from a theorem of Wigner $[1,13]$ that the representatives $\Lambda_{s, t}$ can be chosen in such a way that the map $(s, t) \rightarrow \Lambda_{s, t}$ is strongly separately continuous. This choice is clearly non-unique only to the extent of multiplying $\Lambda_{s, t}$ by $\alpha(s, t)$ and $\Lambda_{s, t}^{\prime}$ by $\bar{\alpha}(s, t)$, where $\alpha$ is a separately continuous $\boldsymbol{T}$-valued function. 
Theorem 6.1. There exists a unique system of representatives $\Lambda_{s, t}$ of the unitary rays $\boldsymbol{\Lambda}_{s_{1},}, s \geq t$, such that

a) $\left(\Lambda_{s, t}: s \geq t\right)$ is a (strongly continuous) unitary evolution, that is, for $r \geq s \geq t$

$$
\Lambda_{r, s} \Lambda_{s, t}=\Lambda_{r, t} .
$$

b) For arbitrary $r \in \boldsymbol{R}$ and $s \geq t$

$$
\Gamma\left(I \oplus S_{r}\right) \Lambda_{s, t} \Gamma\left(I \oplus S_{r}\right)^{-1}=\Lambda_{r+s, r+t}
$$

and

$$
\Gamma(I \oplus R) \Lambda_{s, t}^{*} \Gamma(I \oplus R)^{-1}=\Lambda_{-t,-s}
$$

Proof. The proof consists of three stages of which the first two are lemmas.

Lemma 6.2. There exists a system of representatives $\Lambda_{s, t}, s \geq t$ forming an evolution. Moreover this system is non-unique to the extent of multiplying each $\Lambda_{s, t}$ by $\beta(s, t)$, where the $\boldsymbol{T}$-valued function $\beta$ is separately continuous and satisfies the functional equation

$$
\beta(r, t)=\beta(r, s) \beta(s, t) \quad(r \geq s \geq t) .
$$

Proof of Lemma 6.2. By second quantising the evolution equation satisfied by $\left(U_{s, t}\right)$ we obtain, for $r \geq s \geq t$

$$
\Gamma\left(U_{r, t}\right)=\Gamma\left(U_{r, s}\right) \Gamma\left(U_{s, t}\right) .
$$

For any strongly separately continuous choice of representatives $\left(\Lambda_{s, t}\right)$ we have, on the one hand

$$
\Gamma\left(U_{r, t}\right)=\Lambda_{r, t} \Lambda_{r, t}^{\prime},
$$

where

$$
\Lambda_{r, t}^{\prime}=\Lambda_{r, t}^{-1} \Gamma\left(U_{r, t}\right)
$$

while on the other hand, from (6.6)

$$
\begin{aligned}
\Gamma\left(U_{r, t}\right) & =\Lambda_{r, s} \Lambda_{r, s}^{\prime} \Lambda_{s, t} \Lambda_{s, t}^{\prime} \\
& =\Lambda_{r, s} \Lambda_{s, t} \Lambda_{r, s}^{\prime} \Lambda_{s, t}^{\prime}
\end{aligned}
$$

and $\Lambda_{r, s} \Lambda_{s, t}$ and $\Lambda_{r, s}^{\prime} \Lambda_{s, t}^{\prime}$ are unitary elements of $N_{\sigma}$ and $N_{\sigma}^{\prime}$ respectively. Hence, by the non-uniqueness of the factorisation (6.1) for $\Gamma\left(U_{r . t}\right)$, for some $\alpha(r, s, t) \in \boldsymbol{T}$ we have, for $r \geq s \geq t$, 


$$
\Lambda_{r, t}=\alpha(r, s, t) \Lambda_{r, s} \Lambda_{s, t} .
$$

Clearly the function $\alpha$ is separately continuous in $r, s$ and $t$. By defining $\Lambda_{s, t}=\Lambda_{t, s}^{-1}$ for $s<t$ and extending the domain of definition of $\alpha$ to all of $\boldsymbol{R}^{3}$ by the convention

$$
\alpha(r, s, t)=\left(\alpha\left(r^{\prime}, s^{\prime}, t^{\prime}\right)\right)^{\delta},
$$

where $\delta$ is the sign of the permutation $(r, s, t) \rightarrow\left(r^{\prime}, s^{\prime}, t^{\prime}\right)$, we may assume that (6.7) holds for all $r, s, t \in \mathbb{R}$. From the associativity identity

$$
\left(\Lambda_{r, s} \Lambda_{s, t}\right) \Lambda_{t, 0}=\Lambda_{r, s}\left(\Lambda_{s, t} \Lambda_{t, 0}\right)
$$

we obtain the functional equation for $\alpha$

$$
\alpha(r, s, t) \alpha(r, t, 0)=\alpha(r, s, 0) \alpha(s, t, 0),
$$

that is

$$
\alpha(r, s, t)=\frac{\alpha(r, s, 0) \alpha(s, t, 0)}{\alpha(r, t, 0)},
$$

so that (6.7) can be written

$$
\alpha(r, t, 0) \Lambda_{r, t}=\alpha(r, s, 0) \Lambda_{r, s} \alpha(s, t, 0) \Lambda_{s, t} \text {. }
$$

Replacing each representative $\Lambda_{s, t}$ by $\alpha(s, t, 0) \Lambda_{s, t}$ we thus obtain representatives satisfying the evolution equation (6.2). If $\tilde{\Lambda}_{s, t}=$ $\beta(s, t) \Lambda_{s, t}, s \geq t$, is another set of such representatives then, comparing (6.2) with the evolution equation for $\left(\tilde{\Lambda}_{s, t}\right)$ in the form

$$
\beta(r, s) \Lambda_{r, s} \beta(s, t) \Lambda_{s, t}=\beta(r, t) \Lambda_{r, t}
$$

shows that $\beta$ satisfies (6.5), completing the proof of the lemma.

The general solution of the functional equation (6.5) is easily seen to be

$$
\beta(s, t)=\gamma(s) / \gamma(t)
$$

where the function $\gamma$ must be taken to be continuous to ensure separate continuity of $\beta$, and is unique if it is required to satisfy

$$
\gamma(0)=1 \text {. }
$$

Lemma 6.3. There exists a system of representatives $\Lambda_{s, t}, s \geq t$ forming an evolution and in addition satisfying (6.3). Moreover this system is non-unique to the extent of multiplying each $\Lambda_{s, t}$ by $e^{i \lambda(s-t)}$, 
where $\lambda$ is a real number.

Proof of Lemma 6.3. Second quantising (1.2) we have, for $r \in \boldsymbol{R}$, $s \geq t$

$$
\Gamma\left(I \oplus S_{r}\right) \Gamma\left(U_{s, t}\right) \Gamma\left(I \oplus S_{r}\right)^{-1}=\Gamma\left(U_{r+s, r+t}\right)
$$

and hence, choosing representatives of each $\Gamma\left(U_{s, t}\right)$ in accordance with Lemma 1

$$
\begin{aligned}
\Gamma\left(U_{s, t}\right) & =\Gamma\left(I \oplus S_{r}\right)^{-1} \Gamma\left(U_{r+s, r+t}\right) \Gamma\left(I \oplus S_{r}\right) \\
& =\Gamma\left(I \oplus S_{r}\right)^{-1} \Lambda_{r+s, r+t} \Lambda_{r+s, r+t}^{\prime} \Gamma\left(I \oplus S_{r}\right) \\
& =\Gamma\left(I \oplus S_{r}\right)^{-1} \Lambda_{r+s, r+t} \Gamma\left(I \oplus S_{r}\right) \Gamma\left(I \oplus S_{r}\right)^{-1} \Lambda_{r+s, r+t}^{\prime} \Gamma\left(I \oplus S_{r}\right) .
\end{aligned}
$$

Now by (4.9) and (4.10), conjugation by the second quantisation

$$
\Gamma\left(I \oplus S_{r}\right)^{-1}=\Gamma\left(I \oplus S_{r}^{-1}\right)
$$

is an automorphism of both $N_{\sigma}$ and $N_{\sigma}^{\prime}$. It follows that (6.10) gives a factorisation of $\Gamma\left(U_{s, t}\right)$ into unitary operators

$$
\begin{aligned}
& \tilde{\Lambda}_{s, t}=\Gamma\left(I \oplus S_{r}\right)^{-1} \Lambda_{r+s, r+t} \Gamma\left(I \oplus S_{r}\right), \\
& \tilde{\Lambda}_{s, t}^{\prime}=\Gamma\left(I \oplus S_{r}\right)^{-1} \Lambda_{r+s, r+t}^{\prime} \Gamma\left(I \oplus S_{r}\right)
\end{aligned}
$$

in $N_{\sigma}$ and $N_{\sigma}^{\prime}$ respectively, moreover the representatives $\tilde{\Lambda}_{s, t}$ clearly inherit strong separate continuity. Moreover, since the original representatives formed an evolution, we have, for $u \geq s \geq t$

$$
\begin{aligned}
\tilde{\Lambda}_{u, s} \tilde{\Lambda}_{s, t} & =\Gamma\left(I \oplus S_{r}\right)^{-1} \Lambda_{r+u, r+s} \Gamma\left(I \oplus S_{r}\right) \Gamma\left(I \oplus S_{r}\right)^{-1} \Lambda_{r+s, r+t} \Gamma\left(I \oplus S_{r}\right) \\
& =\Gamma\left(I \oplus S_{r}\right)^{-1} \Lambda_{r+u, r+s} \Lambda_{r+s, r+t} \Gamma\left(I \oplus S_{r}\right) \\
& =\Gamma\left(I \oplus S_{r}\right)^{-1} \Lambda_{r+u, r+t} \Gamma\left(I \oplus S_{r}\right) \\
& =\tilde{\Lambda}_{u, t},
\end{aligned}
$$

that is the new representatives also form an evolution. It follows from Lemma 1, together with the form (6.8), (6.9) of the solutions to the equation (6.5) that, for each $r \in \boldsymbol{R}$, there is a continuous function $\gamma_{r}: \boldsymbol{R} \rightarrow \boldsymbol{T}$ with $\gamma_{r}(0)=1$ such that

$$
\Gamma\left(I \oplus S_{r}\right)^{-1} \Lambda_{r+s, r+t} \Gamma\left(I \oplus S_{r}\right)=\frac{\gamma_{r}}{\gamma_{r}}(t) \frac{(s)}{s, t}
$$

or equivalently

$$
\Lambda_{r+s, r+t}=\frac{\omega(r, s)}{\omega(r, t)} \Gamma\left(I \oplus S_{r}\right) \Lambda_{s, t} \Gamma\left(I \oplus S_{r}\right)^{-1}
$$

where we have set

$$
\omega(s, t)=\gamma_{s}(t)
$$


Comparing the two expressions

$$
\begin{aligned}
& \Lambda_{r+v+s, r+v+t}=\frac{\omega(r+v, s)}{\omega(r+v, t)} \Gamma\left(I \oplus S_{r+v}\right) \Lambda_{s, t} \Gamma\left(I \oplus S_{r+v}\right)^{-1}, \\
& \Lambda_{r+v+s, r+v+t}=\frac{\omega(r, v+s)}{\omega(r, v+t)} \Gamma\left(I \oplus S_{r}\right) \Lambda_{v+s, v+t} \Gamma\left(I \oplus S_{r}\right)^{-1} \\
& =\frac{\omega(r, v+s) \omega(v, s)}{\omega(r, v+t) \omega(v, t)} \Gamma\left(I \oplus S_{r}\right) \Gamma\left(I \oplus S_{v}\right) \Lambda_{s, t} \Gamma\left(I \oplus S_{v}\right)^{-1} \Gamma\left(I \oplus S_{r}\right)^{-1} \\
& =\frac{\omega(r, v+s) \omega(v, s)}{\omega(r, v+t) \omega(v, t)} \Gamma\left(I \oplus S_{r+v}\right) \Lambda_{s, t} \Gamma\left(I \oplus S_{r+v}\right)^{-1}
\end{aligned}
$$

shows that, for arbitrary $r, v \in \boldsymbol{R}$ and $s \geq t$

$$
\frac{\omega(r+v, s)}{\omega(r+v, t)}=\frac{\omega(r, v+s) \omega(v, s)}{\omega(r, v+t) \omega(v, t)} .
$$

Setting $t=0$ in $(6.13)$ and using the fact that $\omega(., 0) \equiv 1$ gives

$$
\omega(r+v, s) \omega(r, v)=\omega(r, v+s) \omega(v, s)
$$

for arbitrary $s \geq 0$, while setting $s=0$ gives

$$
\omega(r, v+t) \omega(v, t)=\omega(r+v, t) \omega(r, v)
$$

for arbitrary $t \leq 0$. Hence $\omega$ satisfies the multiplier equation (6.14) for arbitrary $r, v, s \in \boldsymbol{R}$. In addition $\omega(\cdot, 0) \equiv 1$. Setting $t=0$ in (6.11) shows that $\omega$ is measurable. Every measurable solution of the multiplier equation (6.14) is of form

$$
\omega(s, t)=\frac{\delta(s) \delta(t)}{\delta(s+t)}
$$

for some $\delta: \boldsymbol{R} \rightarrow \boldsymbol{T}$ with $\delta(0)=1$; in particular $\omega$ is symmetric. From (6.12) $\omega$ is continuous in its second argument, hence $\omega$ is separately continuous in both arguments. But then, [8], the function $\delta$ can be chosen to be continuous. Substituting the solution (6.15) into (6.11) we have

$$
\Lambda_{r+s, r+t}=\frac{\delta(s) \delta(r+t)}{\delta(t) \delta(r+s)} \Gamma\left(I \oplus S_{r}\right) \Lambda_{s, t} \Gamma\left(I \oplus S_{r}\right)^{-1}
$$

Hence the system of representatives

$$
\tilde{\Lambda}_{s, t}=\frac{\delta(s)}{\delta(t)} \Lambda_{s, t}
$$

satisfies

$$
\tilde{\Lambda}_{r+s, r+t}=\Gamma\left(I \oplus S_{r}\right) \tilde{\Lambda}_{s, t} \Gamma\left(I \oplus S_{r}\right)^{-1},
$$

which is equivalent to the validity of (6.3). Since $\delta$ is determined 
by (6.15) non-uniquely to the extent of multiplication by a continuous solution $\eta$ of the functional equation

$$
\eta(s) \eta(t)=\eta(s+t), \quad \eta(0)=1
$$

that is by a function of form

$$
\eta(t)=e^{i \lambda t}
$$

for some $\lambda \in \boldsymbol{R}$, it is clear that the new system of representatives is non-unique to the extent of multiplying each $\tilde{\Lambda}_{s, t}$ by $e^{i \lambda(s-t)}$. This completes the proof of Lemma 2 .

To complete the proof of the theorem we must show that there is a unique choice of $\lambda$ such that, for the system of representatives $\Lambda_{s, t}$ of Lemma 2, the system $e^{i \lambda(s-t)} \Lambda_{s, t}$ will satisfy (6.4). By second quantising the relation (1.3) we have

$$
\Gamma(I \oplus R) \Gamma\left(U_{s, t}\right) * \Gamma(I \oplus R)^{-1}=\Gamma\left(U_{-t,-s}\right)
$$

or equivalently

$$
\begin{aligned}
\Gamma\left(U_{s, t}\right) & =\Gamma(I \oplus R)^{-1} \Gamma\left(U_{-t,-s}\right) * \Gamma(I \oplus R) \\
& =\Gamma(I \oplus R)^{-1}\left(\Lambda_{-t,-s} \Lambda_{-t,-s}^{\prime}\right) * \Gamma(I \oplus R) \\
& =\Gamma(I \oplus R)^{-1} \Lambda_{-t,-s}^{\prime *} \Lambda_{-t,-s}^{*} \Gamma(I \oplus R) \\
& =\Gamma(I \oplus R)^{-1} \Lambda_{-t,-s}^{*} \Lambda_{-t,-s}^{\prime *} \Gamma(I \oplus R) \\
& =\tilde{\Lambda}_{s, t} \tilde{\Lambda}_{s, t}^{\prime},
\end{aligned}
$$

where

$$
\begin{aligned}
& \tilde{\Lambda}_{s, t}=\Gamma(I \oplus R)^{-1} \Lambda_{-t,-s}^{*} \Gamma(I \oplus R), \\
& \tilde{\Lambda}_{s, t}^{\prime}=\Gamma(I \oplus R)^{-1} \Lambda_{-t_{,}-s}^{\prime *} \Gamma(I \oplus R)
\end{aligned}
$$

are elements of $N_{\sigma}, N_{\sigma}^{\prime}$ respectively. $\tilde{\Lambda}_{s, t}$ clearly inherits separate continuity in $s$ and $t$ from $\Lambda_{s, t}$. Moreover for $r \geq s \geq t$,

$$
\begin{aligned}
\tilde{\Lambda}_{r, s} \tilde{\Lambda}_{s, t} & =\Gamma(I \oplus R)^{-1} \Lambda_{-s,-r}^{*} \Lambda_{-t,-s}^{*} \Gamma(I \oplus R) \\
& =\Gamma(I \oplus R)^{-1}\left(\Lambda_{-t,-s} \Lambda_{-s,-r}\right) * \Gamma(I \oplus R) \\
& =\Gamma(I \oplus R)^{-1} \Lambda_{-t,-r}^{*} \Gamma(I \oplus R) \\
& =\tilde{\Lambda}_{r, t},
\end{aligned}
$$

so that the representatives $\tilde{\Lambda}_{s, t}$ form an evolution, and for $r \in \boldsymbol{R}$, $s \geq t$,

$$
\begin{aligned}
\Gamma & \left(I \oplus S_{r}\right) \tilde{\Lambda}_{s, t} \Gamma\left(I \oplus S_{r}\right)^{-1} \\
\quad & =\Gamma\left(I \oplus S_{r}\right) \Gamma(I+R)^{-1} \Lambda_{-t,-s}^{*} \Gamma(I \oplus R) \Gamma\left(I \oplus S_{r}\right)^{-1} \\
& =\Gamma\left(I \oplus S_{r} R^{-1}\right) \Lambda_{-t,-s}^{*} \Gamma\left(I \oplus S_{r} R^{-1}\right)^{-1}
\end{aligned}
$$




$$
\begin{aligned}
& =\Gamma\left(I \oplus R^{-1} S_{-r}\right) \Lambda_{-t,-s}^{*} \Gamma\left(I \oplus R^{-1} S_{-r}\right)^{-1} \\
& =\Gamma(I \oplus R)^{-1} \Gamma\left(I \oplus S_{-r}\right) \Lambda_{-t,-s}^{*} \Gamma\left(I \oplus S_{-r}\right)^{-1} \Gamma(I \oplus R) \\
& =\Gamma(I \oplus R)^{-1}\left[\Gamma\left(I \oplus S_{-r}\right) \Lambda_{-t,-s} \Gamma\left(I \oplus S_{-r}\right)^{-1}\right]^{*} \Gamma(I \oplus R) \\
& =\Gamma(I \oplus R)^{-1}\left[\Lambda_{-r-t,-r-s}\right]^{*} \Gamma(I \oplus R) \\
& =\tilde{\Lambda}_{r+s, r+t}
\end{aligned}
$$

Hence the representatives $\tilde{\Lambda}_{s, t}$ also satisfy (6.3). It follows that there is a real number $\mu$ such that, for all $s \geq t$,

$$
\tilde{\Lambda}_{s, t}=e^{i \mu(s-t)} \Lambda_{s, t}
$$

that is

$$
e^{i \mu(s-t)} \Lambda_{s, t}=\Gamma(I \oplus R)^{-1} \Lambda_{-t,-s}^{*} \Gamma(I \oplus R) .
$$

Setting $\mu=2 \lambda$ we find that the system of representatives $e^{i \lambda(s-t)} \Lambda_{s, t}$ satisfies (6.4) as required. It is clear that the choice of $\lambda$ is unique.

\section{§7. The Finite Temperature Boson Feynman-Kac Formula}

Let

$$
A_{t}=e^{-t H}, \quad t \geq 0
$$

be a strongly continuous self-adjoint contraction semigroup acting in the Hilbert space $\mathfrak{h}_{0}$, with trace-class infinitesimal generator $H$, and let $\left(U_{s, t}: s \geq t\right)$ be its time-orthogonal unitary dilation acting in $\mathfrak{h}_{1}=\mathfrak{h}_{0} \oplus \mathfrak{h}$ where $\mathfrak{h}=L^{2}\left(\boldsymbol{R} ; \mathfrak{h}_{0}\right)$. Fix $\sigma>1$. For $s>t$ write

$$
\Gamma\left(\mathfrak{h}_{1}\right)=\Gamma\left(\mathfrak{h}_{0} \oplus \mathfrak{h}_{1 t, s]}\right) \otimes \Gamma\left(\mathfrak{h}_{j l, s]}^{\perp}\right)
$$

corresponding to the direct sum decomposition

$$
\mathfrak{h}_{1}=\left(\mathfrak{h}_{0} \oplus \mathfrak{h}_{] t, s]}\right) \oplus \mathfrak{h}_{\mathfrak{J}, s]}^{\perp}
$$

and let

$$
\begin{gathered}
N_{s, t}=N_{\sigma}\left(\mathfrak{h}_{0} \oplus \mathfrak{h}_{] t, s]}\right) \otimes I, \\
N=N_{\sigma}\left(\mathfrak{h}_{1}\right) .
\end{gathered}
$$

Then, because the map $f \rightarrow W_{\sigma}(f)$ inherits strong continuity from the corresponding Fock representation and because $\cup_{s>t} \mathfrak{h}_{\exists t, s]}$ is dense in $\mathfrak{h},\left(N_{s, t}: s \geq t\right)$ is a double filtration of $N$. Moreover by (4.9) the conjugations by $\Gamma\left(I \oplus S_{r}\right), r \in \boldsymbol{R}$ and by $\Gamma(I \oplus R)$ are automorphisms $\gamma_{r}$ and $\rho$ of $N$, and since $S_{r} \mathfrak{h}_{] t, s]} \subseteq \mathfrak{h}_{] t+r, s+r]}$ and $R \mathfrak{h}_{] t, s]} \subseteq \mathfrak{h}_{3-s,-t]}$ these 
obey (1.1) and (1.2). Thus $\left(N,\left(N_{s, t}\right),\left(\gamma_{t}\right), \rho\right)$ is a reflective covariant system.

Next, let $j$ be the vacuum conditional expectation from $N$ into $B\left(\Gamma\left(\mathfrak{h}_{0}\right)\right)$, so that, for $T \in N, j(T)$ is the unique element of $B\left(\Gamma\left(\mathfrak{h}_{0}\right)\right)$ such that, for arbitrary $\phi, \chi \in \Gamma\left(\mathfrak{h}_{0}\right)$

$$
\langle\psi, j(T) \chi\rangle=\langle\phi \otimes \Omega(\mathfrak{h}), T \chi \otimes \Omega(\mathfrak{h})\rangle .
$$

In fact $j$ maps $N$ into $N_{\sigma}\left(\mathfrak{h}_{0}\right)$; since finite linear combinations of the operators $W_{o}(\xi, f), \xi \in \mathfrak{h}_{0}, f \in \mathfrak{h}$ are strongly dense in $N$ it is sufficient, to see this, to observe that

$$
j\left(W_{\sigma}(\xi, f)\right)=e^{-\frac{\sigma^{2}}{4}\|f\|^{2}} W_{\sigma}(\xi)
$$

as follows from the tensor decomposition

$$
W_{\sigma}(\xi, f)=W_{\sigma}(\xi) \otimes W_{\sigma}(f)
$$

which in turn follows easily from the corresponding decomposition property of the Fock Weyl operators. Since for $f \in \mathfrak{h}_{] t, s]}, g \in \mathfrak{h}_{] u, r]}$ with ]$t, s],] u, r]$ disjoint

$$
\begin{aligned}
j\left(W_{\sigma}(\xi, f) W_{\sigma}(\eta, g)\right) & =\exp \left(-\frac{1}{2} i\langle(\xi, f),(\eta, g)\rangle j\left(W_{\sigma}(\xi+\eta, f+g)\right)\right. \\
& =\exp \left(-\frac{1}{2} i\langle\xi, \eta\rangle\right) e^{-\frac{\sigma^{2}}{4}\|f+g\|^{2}} W_{\sigma}(\xi+\eta) \\
& =e^{\left.-\frac{\sigma^{2}}{4}\|f\|^{2}+\|g\|^{2}\right)} W_{\sigma}(\xi) W_{\sigma}(\eta) \\
& =j\left(W_{\sigma}(\xi, f)\right) j\left(W_{\sigma}(\eta, g)\right),
\end{aligned}
$$

a similar density argument shows that $j$ has the property (1.4). Properties (1.3) and (1.5) are immediate, hence $j$ is a reflective covariant reducing map for the system $\left(N,\left(N_{s, t}\right),\left(\gamma_{t}\right), \rho\right)$.

Now let $\left(\Lambda_{s, t}: s \geq t\right)$ be the system of representatives of Theorem 6.1 .

Theorem 7.1. $\left(\Lambda_{s, t}: s \geq t\right)$ is a reflectively covariantly adapted evolution for the reflective covariant system $\left(N,\left(N_{s, t}\right),\left(\gamma_{t}\right), \rho\right)$.

Proof. We need only prove that $\Lambda_{s, t} \in N_{s, t}$; everything else is a consequence of Theorem 6.1. Writing $U_{s, t}$ as $V_{s, t} \oplus I$ for $V_{s, t}$ acting in $\mathfrak{h}_{0} \oplus \mathfrak{h}_{] t, s]}$, we have from (4.6)

$$
\Gamma\left(U_{s, t}\right)=\Gamma\left(V_{s, t}\right) \otimes I .
$$


The unitary operator $V_{s, t}$ inherits from $U_{s, t}$ the property of differing from the identity by a Hilbert-Schmidt operator. It follows that $\Gamma\left(V_{s, t}\right)$ can be factorised as

$$
\Gamma\left(V_{s, t}\right)=\Lambda \Lambda^{\prime}
$$

for

$$
\Lambda \in N_{\sigma}\left(\mathfrak{h}_{0} \oplus \mathfrak{h}_{], s]}\right), \quad \Lambda^{\prime} \in N_{\sigma}^{\prime}\left(\mathfrak{h}_{0} \oplus \mathfrak{h}_{3 t, s]}\right)
$$

Hence also

$$
\Gamma\left(U_{s, t}\right)=(\Lambda \otimes I)\left(\Lambda^{\prime} \otimes I\right) .
$$

Now

$$
\Lambda \otimes I \in N_{\sigma}\left(\mathfrak{h}_{0} \oplus \mathfrak{h}_{\left.\mathfrak{J}_{t, . s}\right]}\right) \otimes I \subseteq N_{\sigma}\left(\mathfrak{h}_{1}\right)
$$

and similarly $\Lambda^{\prime} \otimes I \in N_{\sigma}^{\prime}\left(\mathfrak{h}_{1}\right)$. Hence by the uniqueness of factorisation of $\Gamma\left(U_{s, t}\right), \Lambda_{s, t}$ is a scalar multiple of $\Lambda \otimes I$. But in that case $\Lambda_{s, t}$ also belongs to $N_{\sigma}\left(\mathfrak{h}_{0} \oplus \mathfrak{h}_{3 t, s]}\right) \otimes I=N_{s, t}$ as required.

\section{References}

[1] Bargmann, V., On unitary ray representations of continuous groups, Ann. of Math (2) 59 (1954), 1-46.

[2] Bratteli, O. and Robinson, D. W., Operator algebras and quantum statistical mechanics II, Springer, Berlin, 1981.

[3] Chevalley, C., The construction and study of certain important algebras, Publ. Math. Soc. Japan 1955.

[4] Evans, D., Completely positive quasi-free maps on the CAR algebra, Commun. Math. Phys. 70 (1979), 53-68.

[5] Hudson, R. L. and Ion, P.D.F., The Feynman-Kac formula for a canonical Boson Wiener process, in Random Fields, ed. J. Fritz et al, North Holland, Amsterdam, 1981.

[6] Hudson, R. L., Ion, P. D. F. and Parthasarathy, K. R., Time-orthogonal unitary dilations and noncommutative Feynman-Kac formulae, Commun. Math. Phys. 83 (1982), 761-80.

[7] The Feynman-Kac formula for Boson Wiener processes, in Quantum Mechanics in Mathematics, Chemistry and Physics, Gustafson, K. (ed), New York, Plenum Press, 1981.

[8] Mackey, G. W., Unitary representations of group extensions, Acta Math. 99 (1958), 265-311.

[9] Schwartz, J., $W^{*}$-algebras, Nelson, London, 1967.

[10] Segal, I. E., Mathematical characterization of the physical vacuum, Ill. J. Math. 6 (1962), 500-23.

[11] Shale, D., Linear symmetries of free Boson fields, Trans. Amer. Math. Soc. 103 (1962), 149-67.

[12] Simon, B., Functional Integration and Quantum Physics, Academic Press, New York, 1979.

[13] Wigner, On unitary representations of the inhomogeneous Lorentz group, Ann. of Math. 40 (1939), 149-204. 
